\title{
A Personalized e-Learning System Based on GWT
}

\author{
Ye Jun Zhanga and Bo Songb + \\ Software College, Shenyang Normal University, Shenyang 110034, China \\ ayejun1108@126.com, bsongbo63@aliyun.comemail
}

Keywords: GWT; Web 2.0; Personalized; E-Learning; Framework.

\begin{abstract}
With the development of Internet technology, information resource is being spread widely, thus the issue of information overload formed. It becomes more difficult for users to retrieve their needed information from so enormous information space. To solve the issue of information overload, recommender system emerges as the times require. This paper presents the personalized e-Learning system using Collaborative Filtering Recommender Algorithm based on clustering of items. And the system will be implemented based on GWT with the advantages that developers can use only one language to develop web application. By the solution of GWT, the function of personalized information recommendation of e-Learning system will be achieved and personalized information will be recommended to users to help them improve learning efficiency. The results show that the solution of GWT is more flexible and simple, reduce the development cycle and cost, improved the real-time requirement of e-Learning system.
\end{abstract}

\section{Introduction}

With the development of Internet technology, huge information resources are presented to us constantly and are being spread widely. The scale of information resources in the internet is also growing, thus the issue of information overload formed. It becomes more difficult for users to browse all of the information resources and find out what they need or they are interested in. Recommender system is one of the solutions to facing information overload issue. It is an intelligent and personalized information service system and describes the user's long-term information need by user modeling, based on which it can customize the personalized information with the specific recommendation strategy [1].

In this paper, we will construct a personalized e-Learning system using Collaborative Filtering Recommendation Algorithm based on clustering of items and will implement the system based on GWT (Google Web Toolkit). For e-Learning system, the main benefit of Ajax is a greatly improved user experience. Although JavaScript and DHTML - the technical foundations of Ajax have been available for years, most programmers ignored them because they were difficult to master. So, if you're planning to use Ajax to improve only your application's user experience - if you're not also using it as a strategic advantage for your business - it may be unwise to spend a lot of money and time on the technology [2]. Aiming at the problems, the application framework of e-Learning system in this paper is implemented based on GWT which is a framework that developers use one language to develop web application. The project uses Java as a single programming language for both the client and server sides. According to the integration of GWT and Spring MVC, not only are the cost and complexity of e-Learning system reduced but the human-computer interaction experience and real-time requirements are improved effectively.

\section{GWT and Spring MVC}

Google offers a new framework named GWT to help Java developers create Ajax applications more productively. It uses Java as a single programming language for both the client and server sides. The

\footnotetext{
+ Corresponding author. Tel.: +861-394-0536713; fax: +86-024-86592390.

E-mail address: songbo63@aliyun.com.
} 
GWT eliminates the complexity of hand coding HTML, JavaScript, and other elements that bind the JavaScript actions triggered in an HTML page (client-side code run within the user's browser) to the controlling layer executing within the Java EE application server. When developers developed within a good IDE, the GWT can provide an immeasurable advantage over other frameworks and considerably lower the cost and complexity of the project.

Spring MVC integrate in Spring Web Flow. Spring MVC separates the controller, the model object, dispatcher and the role of handler object. This separation makes them easier to customize. MVC is the core part of the WEB project development, as the decomposition of the three words, $\mathrm{C}$ (Controller) separate V (View, Client) and M (model, business) constitute MVC. The Spring annotations will make our work easier, by using the reflection mechanism of annotation to solve the problem of a large number of configuration.

In the design of the architecture, the business developer only needs to develop five types of code: UIController, UIWidget, Action, DAO and Entity. UI using the GWT framework provides the UI development. The UI management components have cache management. It is mainly responsible for rendering and input control interface, and it does not call the background service. Besides it is mainly responsible for interactions with the data of the background services, management and scheduling Widget component.

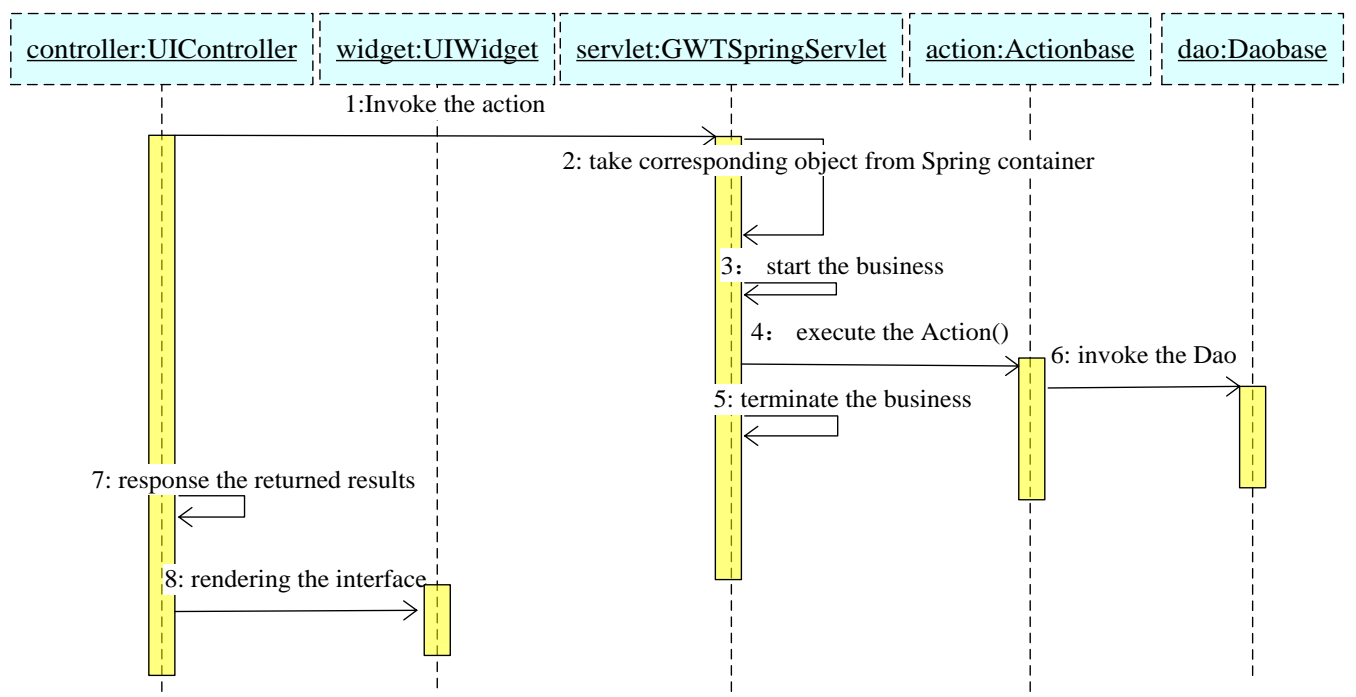

Fig. 1 GWT integration with Spring framework of the e-Learning system.

"Action components" provides the business logic implementation."GWTSpring integration components" will implement GWT to integrate with Spring framework. In the Fig. 1, GWTSpringServlet is "GWTSpring integration components", as is a WEB application service unified listening Servlet, UIController calling back-end services are implemented by GWTSpringServlet route. GWTSpringServlet contains calls to the Spring container packaging, in a WEB application server startup and read the Spring configuration file, to initialize the Spring container. GWTSpringServlet is "GWTSpringintegration components", it is a unified listening Servlet in WEB application service, UIController calls to back-end services are made by GWTSpringServlet route and transmit. Before the call Action method, the Spring container will determine to start a transaction according to the configuration [10].

\section{Recommender system related work}

Personalized recommendation is an important part in user behavior analysis. In simple term, it is a process of searching the resource which the user might interest in [4,5]. At present, Collaborative Filtering is a relatively mature and popular recommendation algorithm. In collaborative filtering, an item is considered as a black box we don't look at its content and user interactions (such as rating, saving, purchasing) with the item are used to recommend an item of interest to the user [6]. More formally, given a dataset of user actions also called the user-item dataset, we will recommend items to 
a current user. The main idea of Collaborative Filtering is to exploit information about the past behavior or opinions of an existing user community for predicting which items the current user will most probably like or be interested in. Collaborative Filtering approaches take a matrix of given user-item ratings as the only input and typically produce the following types of output: a numerical prediction indicating to what degree the current user will like or dislike a certain item. Such a top-N list should not contain items that the current user has already rated [7].

Jacobian measure. Jacobian measure is used in this topic, the consistency on the user ratings represent the intersection of two user ratings set. The total score represents the union set of two score set. The similar result is stored as a similarity matrix it is a two dimensional double type array of:

similarity Values $[\mathrm{u}][\mathrm{v}]=\frac{(\text { double }) \text { agreementCount }}{\text { (double) totalCount }}$

We need to measure the similarity among items in the process of clustering and search the nearest neighbor item. Take the computing similarity of items as an example, the connectivity for the two items $\mathrm{i}$ and $\mathrm{j}$ is to choose a common score users from grade or other user preference matrix and then compute the similarity $S_{i}$, j of the two common score vector. The commonly used three similarity calculation formulas are following: Pearson correlation coefficient, cosine similarity.

Pearson Correlation Coefficient. Pearson coefficient is a kind of similarity calculation method based on correlation coefficient. It needs find the users with common scores, in order to make the accurate calculation results. $U$ is the user set which rate item $i$ and item $j$, the Pearson Correlation Coefficient formula is as follows:

$$
\operatorname{sim}(i, j)=\frac{\sum_{u \in U}\left(R_{u, i}-\bar{R}_{i}\right)\left(R_{u, j}-\bar{R}_{j}\right)}{\sqrt{\left(\sum_{u \in U} R_{u, i}-\bar{R}_{i}\right)^{2}} \sqrt{\left(\sum_{u \in U} R_{u, j}-\bar{R}_{j}\right)^{2}}}
$$

$R_{u, i}$ is the rating given by user u to an item $\mathrm{i}, R_{i}$ is the rating given by user set $\mathrm{U}$ to the same item.

Cosine Similarity. Item i, $\mathrm{j}$ are regard as two n-dimensional vector and estimate the similarity of the two items by calculate the two vector space angle. For the $m^{*} n$ score matrix, the similarity of item $i$ and item $j$ calculation formula as is shown below:

$$
\operatorname{sim}(i, j)=\cos (\vec{i}, \vec{j}) \frac{\vec{i} \cdot \vec{j}}{|\vec{i}| \cdot|\vec{j}|}
$$

In this formula " • " represent the vector inner-product. This is a common formula for estimating the vector similarity. Items were clustered based on users' ratings on items, each cluster has a cluster center. Based on the similarity between target item and cluster centers, the nearest neighbors of target item can be found in the item clusters that most similar to the target item. Preprocessing and clustering the basic user when it offline. Thus it solves the bottleneck problem of generating recommendations speed in the traditional collaborative filtering algorithm and establish more effective recommendation algorithm in the system.

The system uses the K-means algorithm to Cluster the user by the similarity of the score that the user give to the item and put the similar interesting user in the same class. When the user comes, firstly, cluster the user to its cluster. Searching the nearest target user in its cluster, so that improves efficiency to search nearest neighbor of target user. This improves the efficiency greatly and decreases the time finding target items on the whole, as the system does not occupy the online time when it clusters for items offline.

\section{Implementation of the e-Learning system}

As Fig. 2 showed, a kind of hierarchical and extensible e-Learning system is proposed in this paper. In this Fig. 2, the rich client is deployed and run in the user's browser, and the layers of server-side is deployed and run in the Java EE application server, and the communication mode is Ajax between the 
two. The rich client is provided with widget by GWT based on Ajax. The framework proposed by this paper makes full use of the characteristic of GWT technology and obtains further depuration and encapsulation, which enable the framework more suitable for the design and development of specific e-Learning system [10].

In Fig. 2, the GWT services straddle the line between controllers and services, since they basically provide the HTTP processing capability of a controller and perform some GWT serialization work on the results from the service layer. The DAO layer contains the code that produces the results of our business processes persistent and queries the database. Accessing the database directly from within the business methods, we need to access the database when we unit test these services, which will complicate our unit tests considerably. With an injected DAO layer, to inject mock DAO objects instead, it will make unit tests far easier to do.

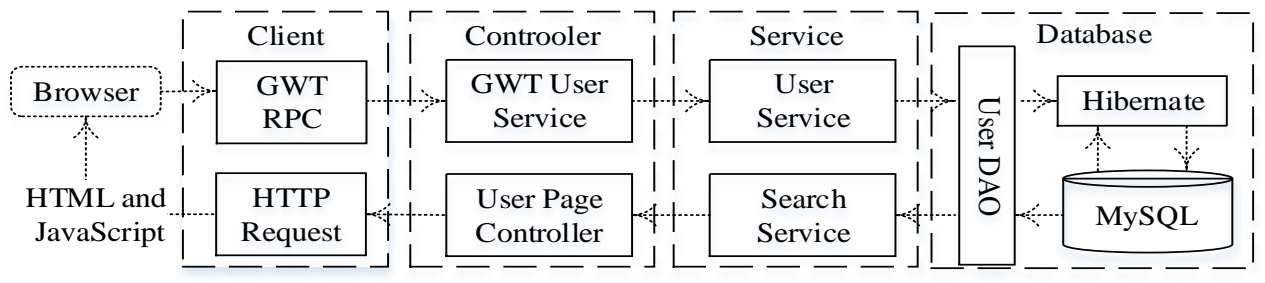

Fig. 2 The Framework of the e-Learning system.

Service calling layer is responsible for calling service offered by the service layer of server-side and returning the result to client logic layer and client presentation layer. In other words, the service calling layer decouple between foreground logic and background logic. According to each YourService in this layer, a YourServiceCaller class is designed. Both the construction of the service object and the access of operating the service object are encapsulating, which enable client-side to call the service by YourServiceCaller class. In doing so, it is implemented to centralized control of background service access in order to maintain the code. The class diagram of the GWT RPC service extended framework about e-Learning system is shown in Fig. 3.

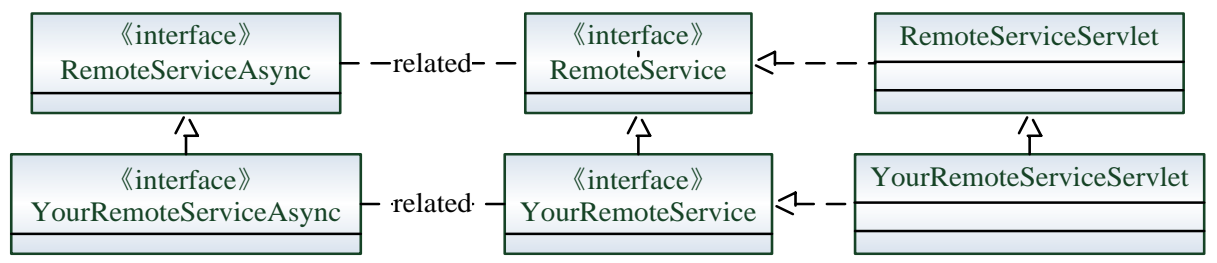

Fig. 3 The Framework of the e-Learning system.

\section{Conclusion}

In the personalized recommender system, the core feature of personalized information is user-cantered and to generate the recommendation items which are available and reflect the preferences of individuals. The recommender system of e-Learning system in this paper is implemented based on GWT. Fig. 4 shows the aggregate average response time (AART) of the system of the application architecture based on open source framework - Struts, Hibernate, Spring (SHS), Java EE and GWT and Spring MVC.

The testing scenario is information query in the program of client-side. When analyzing the AART curve in Fig. 4, compared with the e-Learning system solutions of [7] and [9], we can find that the system response time of the solution proposed by this paper may increase according to the curve until it reaches 100 users. After that time, the increase of the curve changes more acutely. My solution compared with the solution of SHS, the system response time of the solution of Java EE increases in accordance with the linear way until it reaches about 100 users. Through analyzing the changing tendency of curves, in the performance test, the maximum limit on the number of users is 100, so it meets the needs of the application service. The solution of GWT and Spring MVC is close to the 
Solution of Java EE AART. Finally, in this paper how to improve e-Learning system construction will be discussed which has some guiding significance for the application and development of the e-Learning system construction in the network teaching field.

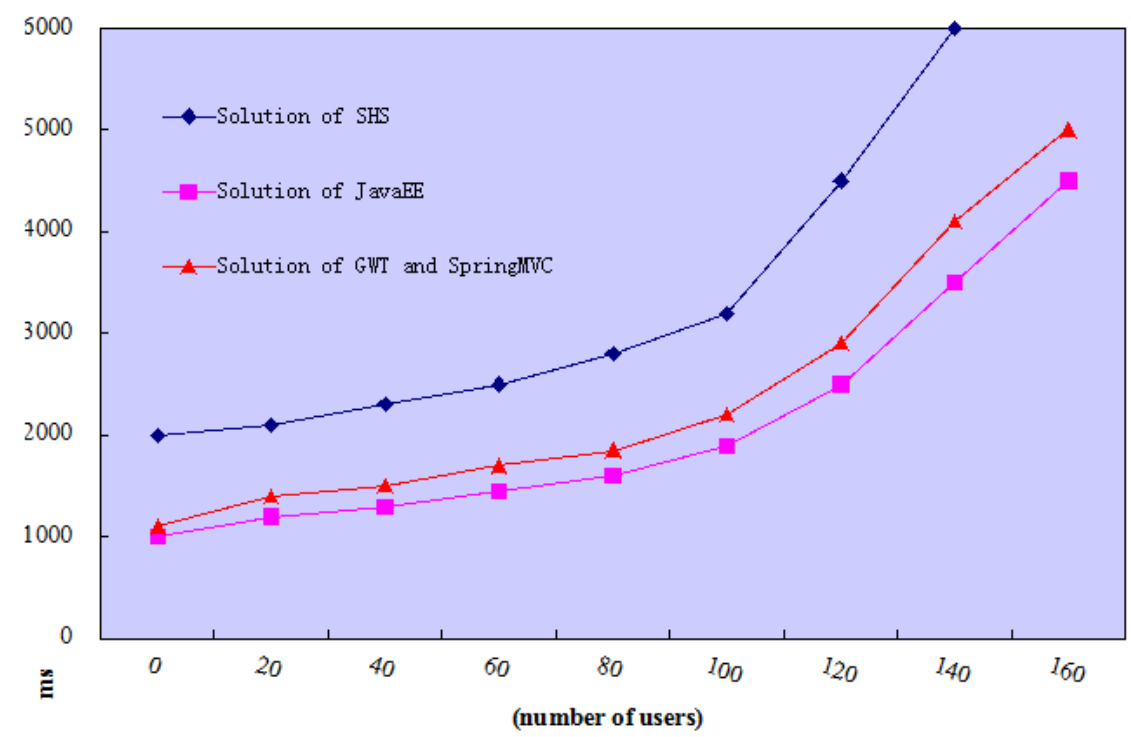

Fig. 4 AART curve.

\section{References}

[1] L. Ren, The Key Technology Research of Recommender System, East China Normal University, 2012.

[2] B. Song and M. Y. Li, “An e-Learning System Based on GWT and Berkeley DB”, Lecture Notes in Computer Science 7332, Vol.2, 2012, pp. 26-32.

[3] J. Xiao and L. He, An expandable recommendation system on IPTV, Lecture Notes in Computer Science 7332, vol. 2, 2012, pp. 33-40.

[4] S. Alag, Collective intelligence in action, Manning Publications, 2009,pp. 349-350.

[5] J.Dietmar, Z.Markus, F.Alexander, and F. Gerhard, Recommender system anintroduction, Cambridge University Press, 2011, pp. 13-19.

[6] B. Song and J. Liu, Implementation of J2EE Data Persistence Tier with TopLink, Microelectronics \& Computer, 23(8), 2006, pp. 132-135.

[7] B. Song and J. Zhao, Research on Network Teaching System Based on Open Source Framework, IEEE Ninth International Conference on Hybrid Intelligent Systems, Vol.1, IEEE: Shenyang, 2009, pp. 28-32.

[8] W. Fang and Y. Sun, Research and Application of J2EE's Data Persistence Layer, Computer Technology and Development, 17(2), 2007, pp. 68-91.

[9] B. Song and Y. Zhang, Implementation on Network Teaching System Based on Java EE Architecture, IEEE Second International Conference on Information Technology and Computer Science, Vol.1, Kiev, 2010, pp. 354-357.

[10]B. Song and Y. Zhang, A e-Learning System Based on GWT and Spring MVC, Transactions on Information and Communication Technologies, 2014, pp.171-179. 